AperTO - Archivio Istituzionale Open Access dell'Università di Torino

\title{
An ecoepidemic model with diseased predators and prey group defense
}

\section{This is the author's manuscript}

Original Citation:

Availability:

This version is available http://hdl.handle.net/2318/145459

since

Published version:

DOI:10.1016/j.simpat.2013.02.004

Terms of use:

Open Access

Anyone can freely access the full text of works made available as "Open Access". Works made available under a Creative Commons license can be used according to the terms and conditions of said license. Use of all other works requires consent of the right holder (author or publisher) if not exempted from copyright protection by the applicable law. 


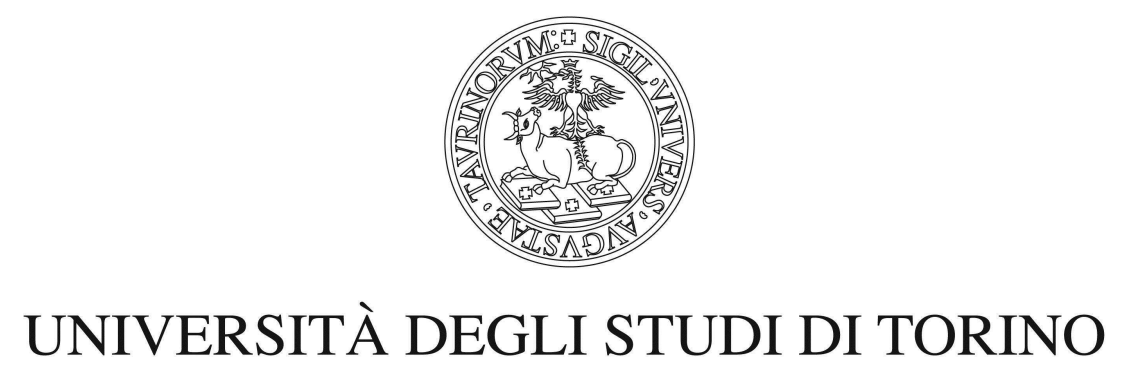

This is an author version of the contribution published on:

Sabrina Belvisi, Ezio Venturino. An ecoepidemic model with diseased predators and prey group defense. SIMPAT, 34, 144-155, 2013. DOI:

10.1016/j.simpat.2013.02.004

The definitive version is available at:

http://ees.elsevier.com/simpat/ 


\title{
An ecoepidemic model with diseased predators and prey group defense
}

\author{
Sabrina Belvisi, Ezio Venturino ${ }^{1}$ \\ Dipartimento di Matematica "Giuseppe Peano", \\ Università di Torino, via Carlo Alberto 10, 10123 Torino, Italy \\ E-mail: ezio.venturino@unito.it
}

\begin{abstract}
In this paper we consider a prey population that gathers in herds, together with its interactions with predators. The latter are affected by a disease, and only the healthy individuals hunt. The defensive attitude of the prey is reflected in the fact that mostly the individuals at the border of the herd suffer from the attacks of the predators. Equilibria and their stability are fully analysed. The system can either eliminate the predators, or the disease can be eradicated, or finally infected and healthy predators can coexist with prey. In particular circumstances persistent population oscillations can arise. The ultimate behavior of the system is characterized by two suitably introduced parameters, one of demographic nature, the other mainly of epidemiological significance. As in other ecoepidemic models, the analysis points out that the disease plays a relevant influence also when group defense is considered, so that the ultimate outcome of the system is not prescribed just by demographic considerations.
\end{abstract}

Keywords: predator-prey, group defense, ecoepidemic systems, ecosystems

\section{Introduction}

Mathematical population theory, which dates back two centuries to the work of the economist Malthus, [1], originally dealt with the study of the

\footnotetext{
${ }^{1}$ This paper was completed and written during a visit of this author at the Max Planck Institut für Physik Komplexer Systeme in Dresden, Germany. The author expresses his thanks for the facilities provided.
} 
dynamics of a single population. This original model was then modified to compensate for its shortcomings, namely the impossible unbounded growth of the population with only limited resources, by the so-called logistic version, [2]. More than a century later through the influence of the works of Lotka and Volterra, [3, 4], scientists began to consider interacting populations, in particular of competing type, [5]. Mathematical epidemiology evolved from its conception at about the same time, in the late 1920's with the works of Kermack, McKendrick and then of von Foerster, [6, 7]. These two disciplines throughout the past century developed on parallel tracks without much communication among them. The fact that diseases are a common fact in nature, coupled with the consideration that most of them spread by contact among healthy individuals and those infected, or through contaminated materials, and the fact that they influence the mortality of the communities among which they rage, prompted scientists to take a more general view of the demographic interactions encompassing other phenomena such as diseases as well. Similarly, epidemiologists started to investigate the spread of diseases in populations that change in time, as their reproduction is a continuous phenomenon, that influences the disease prevalence. Thus toward the turn of the century, epidemiologists began to consider mathematical models of diseases affecting variable size populations, $[8,9,10]$. This step led to a cross fertilization among the two fields, as a number of papers appeared, $[11,12,13,14]$, in which the spread of diseases began to be investigated among populations that in some way or other were intermingling. A new field of research developed, now called ecoepidemiology, see [15] for more details on its development and more references.

In population theory the basic assumption among interacting populations concerns individual encounters. This is generally modelled on a one-to-one basis via the mass action law. However the case of interactions among isolated predators hunting herds of prey has not been considered until very recently. In fact an idea based on the shape of the response function has been proposed quite some time ago, [16]. A more recent investigation hinges on a rather different idea. Namely, models for prey gathering in herds and using group defense, with the most fit, i.e. healthy, animals occupying the edge of the bunch, have been proposed by one of the authors [17, 18] and the idea has been further pursued [19]. A similar idea had been expounded some time ago for predators hunting in groups, [20].

The next natural step was then to turn to ecoepidemiological models. For a rather recent review of the field, see Chapter 7 of [15]. In this context, the 
models generally assume that the sound individuals do not recognize diseasecarriers. But in $[23,24]$, the situation in which the latter are purposely avoided upon recognition by the healthy animals is expressely taken into account. Prey group defense models have thus been extended to the ecoepidemic case, by considering predator-prey interactions in which the epidemics affects the prey, [21]. In this paper we deal with the reverse situation, i.e. when the disease spreads among predators, [25]. Several examples of diseases affecting wild populations are provided in Table 2 of [26]. Such situations can encompass populations living on the ground, in water and in the air. For instance, with reference to the notation introduced in the model, rabies and Sarcoptes spp. affect foxes (Vulpis vulpis); in water we can mention the Phocine Distemper Virus, which affects the common seal (Phoca vitulina) and the striped dolphin (Stenella coeruleoalba), here the prey being fishes, which in some cases clearly gather in schools. For several bird species, common diseases are caused by, e.g. Avian Pox, Newcastle Disease, Influenza, Pasteurella multocida, Apergillus fumigatus and Leukocytozoon simondi, with mainly insects as their prey. The latter also can gather in swarms.

The paper is organized as follows. In the next Section we give a mathematical description of the system and rephrase it in adimensional form. Section 3 discusses the possible equilibria of the system. In Section 4 we analyse their stability, showing that it is completely characterized by a suitably introduced parameter, the relevance of which had been already discovered in $[17,18]$. Section 5 contains the bifurcation analysis. A final discussion concludes the paper.

\section{The model}

Let $\bar{R}(\tau)$ denote the prey population (the name of the variable is a mnemonic shorthand for rabbits, although they do not live in herds), $\bar{F}(\tau)$ (shorthand for foxes) be the healthy predators and $\bar{G}(\tau)$ the diseased ones at time $(\tau)$. We assume that prey reproduce logistically, at intrinsic growth rate $\bar{r}$ and with carrying capacity $\bar{K}$, and are hunted at rate $\bar{a}$ by the healthy predators. Since they gather together in herd, mainly the individuals on its boundary will be mostly affected by the predators attacks. This, following $[17,18]$, is modelled using a square root term for the prey population, accounting for the prey population occupying this portion of the ground.

Now the square root term is used to model the fact that the perimeter of the occupied ground is a one-dimensional measure, compared to the two- 
dimensional one for the area of a surface. Thus the real shape of the herd need not necessarily be circular, as the root term is multiplied by a constant factor $\bar{a}$, that accounts for geometric as well as biologic features. It can be regarded as the product of two factors, the first one taking care of the likely deformation from the circular shape to possibly a different one, the second one expressing the hunting rate of the healthy predators on the prey.

Note that living in herds has a benefit for the whole population, this arising from the first two equations of the model (1) in that the predation term grows following a concave function, and not linearly as it does in standard predator-prey models. Therefore, as the number of prey grows, those that are captured are in proportion less. This is similar to what happens using a Holling-type II response function, but the underlying biological mechanism is totally different, as the Holling-type II response models feeding satiation in the predators.

The predators are disease-aected, thereby as already stated above, partitioned among susceptibles and infected, with transitions from the former to the latter class at rate $\bar{\lambda}$, denoting the disease transmission rate. The disease is assumed to be unrecoverable. The prey $\bar{r}$ represent the only food source for the predators. This is reected in the natural mortality $\bar{m}$ experienced in absence of prey. When the latter are captured, they are converted into new predators via the conversion factor $0<e \leq 1$, which is applied to the term expressing prey capturing. The infected predators are assumed to be too weak to hunt, and therefore they will die at rate $\bar{n}$, expressing the combination of natural and disease-related mortality. The system reads

$$
\begin{array}{r}
\frac{d \bar{R}}{d \tau}=\bar{r}\left(1-\frac{\bar{R}}{K}\right) \bar{R}-\bar{a} \sqrt{\bar{R}} \bar{F} \\
\frac{d \bar{F}}{d \tau}=\bar{F}(-\bar{m}+e \bar{a} \sqrt{\bar{R}}-\bar{\lambda} \bar{G}) \\
\frac{d \bar{G}}{d \tau}=\bar{G}(-\bar{n}+\bar{\lambda} \bar{F}) .
\end{array}
$$

Clearly, the model has an underlying demographic structure on top of which an epidemic of SI (susceptible-infected) type is introduced. In this context, other types of more complicated epidemics could be considered, such as SIR (susceptible-infected-removed) or SIRS (susceptible-infected-removedsusceptible), in case the disease can be overcome and individuals would be subject to relapses. The problem however, for which we avoid these models 
at present, is that the number of classes in which the predator population should be partitioned would increase, and correspondingly would the size of the dynamical system (1) do, thereby complicating the analysis.

As it has already been observed in $[18,21]$, the square root terms upon differentiation entail the possible presence of a singularity. By a suitable change of dependent variables, we can remove the singularity appearing in the Jacobian matrix, when the prey population vanishes. Namely, we set

$$
\sqrt{\bar{R}}=\bar{P}, \quad \frac{d \bar{P}}{d \tau}=\frac{1}{2 \sqrt{\bar{R}}} \frac{d \bar{R}}{d \tau} .
$$

Here, the new variable $P$ can be interpreted as the number of prey on the boundary of the herd. Substituting into (1) we get

$$
\begin{array}{r}
\frac{d \bar{P}}{d \tau}=\frac{\bar{r}}{2}\left(1-\frac{\bar{P}^{2}}{K}\right) \bar{P}-\frac{\bar{a}}{2} \bar{F} \\
\frac{d \bar{F}}{d \tau}=\bar{F}(-\bar{m}+e \bar{a} \bar{P}-\bar{\lambda} \bar{G}) \\
\frac{d \bar{G}}{d \tau}=\bar{G}(-\bar{n}+\bar{\lambda} \bar{F})
\end{array}
$$

Note that in this very step also the nature of the system changes in part. In fact the last term of the first equation in (1) from nonlinear has become linear in the reformulation. We then adimensionalise this system. We define the adimensional time $t=\delta \tau$ and the new dependent variables $P(t)=\alpha \bar{P}(\tau)$, $F(t)=\beta \bar{F}(\tau), G(t)=\gamma \bar{G}(\tau)$ to obtain

$$
\begin{array}{r}
\frac{d P}{d t}=\frac{\bar{r}}{2 \delta}\left(1-\frac{P^{2}}{\alpha^{2} K}\right) P-\frac{\bar{a} \alpha}{2 \beta \delta} F \\
\frac{d F}{d t}=F\left(-\frac{\bar{m}}{\delta}+\frac{e \bar{a}}{\alpha \delta} P-\frac{\bar{\lambda}}{\gamma \delta} G\right) \\
\frac{d G}{d t}=G\left(-\frac{\bar{n}}{\delta}+\frac{\bar{\lambda}}{\beta \delta} F\right)
\end{array}
$$

Finally, we can set

$$
\alpha^{2} K=1, \quad \frac{\bar{r}}{2 \delta}=1, \quad \frac{\bar{a} \alpha}{2 \beta \delta}=\frac{\bar{a}}{\alpha \delta}, \quad \frac{\bar{\lambda}}{\gamma \delta}=\frac{\bar{\lambda}}{\beta \delta} .
$$


Thus we can now define the new adimensional parameters

$$
m=\frac{\bar{m}}{\delta}, \quad n=\frac{\bar{n}}{\delta}, \quad \frac{\bar{a} \alpha}{2 \beta \delta}=\frac{\bar{a}}{\alpha \delta}=a \quad \frac{\bar{\lambda}}{\gamma \delta}=\frac{\bar{\lambda}}{\beta \delta}=\lambda .
$$

The final form of the system is

$$
\begin{array}{r}
\frac{d P}{d t}=\left(1-P^{2}\right) P-a F, \\
\frac{d F}{d t}=F(-m+e a P-\lambda G), \\
\frac{d G}{d t}=G(-n+\lambda F) .
\end{array}
$$

In part following what has been done in [18], let us further introduce the new parameters, which again are dimensionless, since $m, a, h$ and $n$ are, while $e$ is by definition a pure number,

$$
\rho:=\frac{m}{e a}, \quad h:=\frac{a n}{\lambda} .
$$

\section{Equilibria}

The possibile equilibria of the system are: extinction, predator-free, diseasefree and coexistence. They correspond respectively to the always feasible points $E_{0}=(0,0,0), E_{1}=(1,0,0)$ and

$$
E_{2}=\left(\rho, \frac{\rho}{a}\left(1-\rho^{2}\right), 0\right), \quad E_{3}=\left(P_{3}, \frac{h}{a}, \frac{e a}{\lambda}\left(P_{3}-\rho\right)\right),
$$

where the latter can be a multiple equilibrium, since $P_{3}$ can attain at most two different values, as described below. For $E_{2}$ the feasibility condition is immediately found,

$$
\rho<1 .
$$

The coexistence equilibrium $E_{3}$ needs positivity of the third component as feasibility condition, thereby imposing the lower bound on the prey population

$$
P_{3}>\rho,
$$

which is more stringent than the nonnegativity of the first component, namely given just by the value of the prey population $P_{3}$. The value of the prey population $P_{3}$ is obtained as a root of the cubic equation

$$
f(P) \equiv P^{3}-P+h=0 .
$$


This function has a positive value at the origin, $f(0)=h>0$, a minimum at $P_{0}=3^{-\frac{1}{2}}$ and the inflection point at $P=0$. To have a positive intersection with the $P$ axis, we must have $f\left(P_{0}\right) \leq 0$, thus

$$
h \leq \frac{2 \sqrt{3}}{9}
$$

Further, $f(1)=f(0)=h>0$. We can thus conclude that if $(7)$ holds, there are two real positive roots,

$$
P_{3}^{-} \in\left[0, \frac{1}{\sqrt{3}}\right], \quad P_{3}^{+} \in\left[\frac{1}{\sqrt{3}}, 1\right] .
$$

These roots clearly coalesce if $h=2 \sqrt{3} / 9$. In view of (5) and of the fact that both roots are smaller than one, for compatibility we must impose the additional feasibility condition $\rho<1$, thereby obtaining

$$
1 \geq P_{3} \geq \rho
$$

We summarize our findings in the following proposition.

Proposition 1 The system admits the origin, the predator-free, the disease-free and the coexistence with endemic disease equilibria. For the latter two, feasibility is ensured respectively by condition (4) for $E_{2}$ and conditions (5) and (8) for the coexistence.

\section{Local Stability Analysis}

The Jacobian matrix of (2) is

$$
J=\left(\begin{array}{ccc}
1-3 P^{2} & -a & 0 \\
e a F & -m+e a P-\lambda G & -\lambda F \\
0 & \lambda G & -n+\lambda F
\end{array}\right)
$$

At the origin, the eigenvalues of the Jacobian matrix are $1,-m$ and $-n$, implying thus instability for $E_{0}$. At $E_{1}$ we find instead $-n,-2$ and $-m+e a$. To have stability, we must impose

$$
\rho>1
$$


At $E_{2}$ the characteristic equation of the Jacobian matrix in $x$ factors to give one explicit eigenvalue $x_{1}=\lambda a^{-1}\left[\rho\left(1-\rho^{2}\right)-h\right]$, and the two roots of the quadratic equation

$$
x^{2}-x\left(1-3 \rho^{2}\right)+m\left(1-\rho^{2}\right)=0,
$$

namely

$$
x_{2,3}=\frac{1}{2}\left[\left(1-3 \rho^{2}\right) \pm \sqrt{\Delta}\right], \quad \Delta=\left(1-3 \rho^{2}\right)^{2}-4 m\left(1-\rho^{2}\right) .
$$

Imposing that the real parts of these latter eigenvalues of the Jacobian matrix are negative, $\Re\left(x_{2,3}\right)<0$, we find $3 \rho^{2}>1$, which must be combined with negativity of the first eigenvalue of the Jacobian matrix. In summary, stability of $E_{2}$ holds for

$$
3 \rho^{2}>1, \quad h>\rho\left(1-\rho^{2}\right) .
$$

The characteristic equation of the Jacobian matrix $\sum_{n=0}^{3} a_{n} x^{3-n}=0$ for the coexistence equilibrium $E_{3}$ reads

$$
x^{3}-\left(1-3 P_{3}^{2}\right) x^{2}+\operatorname{aex}\left[n\left(P_{3}-\rho\right)+h\right]-\operatorname{aen}\left(1-3 P_{3}^{2}\right)\left(P_{3}-\rho\right)=0 .
$$

We now apply the Routh-Hurwitz criterion, see p. 67 of [22], to find that stability is ensured if

$$
a_{1}>0, \quad a_{1} a_{2}-a_{0} a_{3}>0, \quad a_{3}>0 .
$$

From this, assuming feasibility, the following stability condition is obtained,

$$
P_{3}>\frac{1}{\sqrt{3}}
$$

The function $f(P)=P^{3}-P+h$ has two real positive roots, one in the interval $\left[0, \frac{1}{\sqrt{3}}\right]$, the other one in $\left[\frac{1}{\sqrt{3}}, 1\right]$. The first one, $P_{3}^{-}$, in view of $(12)$ is always unstable, the second one instead, $P_{3}^{+}$, is always locally asymptotically stable, when it exists. Table 1 summarizes our findings.

We have thus shown the following result.

Proposition 2 Extinction of the system (2) is never possible. The predator-free equilibrium is stable if $\rho>1$. The disease-free equilibrium 


\begin{tabular}{|c|c|c|}
\hline $\begin{array}{c}\text { EQUILIBRIUM } \\
\text { POINT }\end{array}$ & $\begin{array}{c}\text { FEASIBILITY } \\
\text { CONDITIONS }\end{array}$ & $\begin{array}{c}\text { STABILITY } \\
\text { CONDITIONS }\end{array}$ \\
\hline$E_{0}=(0,0,0)$ & - & always unstable \\
$E_{1}=(1,0,0)$ & - & $\rho>1$ \\
$E_{2}=\left(\rho, \frac{\rho}{a}\left(1-\rho^{2}\right), 0\right)$ & $\rho<1$ & $h>\rho\left(1-\rho^{2}\right)$, \\
& & $\rho>\frac{1}{\sqrt{3}}$ \\
$E_{3}=\left(P_{3}, \frac{h}{a}, \frac{e a P_{3}-m}{\lambda}\right)$, & $1>P_{3}>\rho$, & \\
where $P_{3}$ solves & $h \leq \frac{2 \sqrt{3}}{9}$, & $P_{3}>\frac{1}{\sqrt{3}}$ \\
$P^{3}-P+h=0$ & \multicolumn{2}{|c}{} \\
\hline
\end{tabular}

Table 1: Summary of feasibility and stability conditions for the equilibria of (2).

is stable if conditions (10) hold. Coexistence with an endemic disease in the predators is ensured by (12).

Remark 1. As a check, we run simulations with the parameter values: $\lambda=0.4, a=0.5, n=0.5, e=0.5$, with $m$ varying in the range [0.31, 0.51]. We find the results of Figures 1 and 2 . In these cases, equilibrium $E_{1}$ is stable, since $\rho>1$.

Remark 2. For the parameters $\lambda=0.5, a=0.5, m=0.2, e=0.5$, with $n$ in the range $[0.41,0.61]$ we have the results of Figures 3 and 4 . In this case $E_{2}$ is stable, since $3^{-1}=0.577<0.8=\rho<1$ and $n=h>\rho\left(1-\rho^{2}\right)=0.288$.

Remark 3. Finally we consider the parameters $\lambda=0.5, a=0.5$, $n=0.4, e=0.5$, with $m \in[0.205,0.305]$, we have the results of Figures 5 and 6 . Now in this situation $E_{2}$ is initially stable, but when $m$ varies $\rho$ changes from values smaller than 1 to values higher than 1 , so that feasibility of $E_{2}$ does not hold anymore. We rather obtain stability of $E_{1}$, thus showing a transcritical bifurcation, which is also apparent from the interchange of feasibility and stability conditions for $E_{1}$ and $E_{2}$, which can be easily seen for $\rho=1$ in Table 1 .

\section{Study of the bifurcations}

In this section we investigate the behavior of the system as parameters change. 

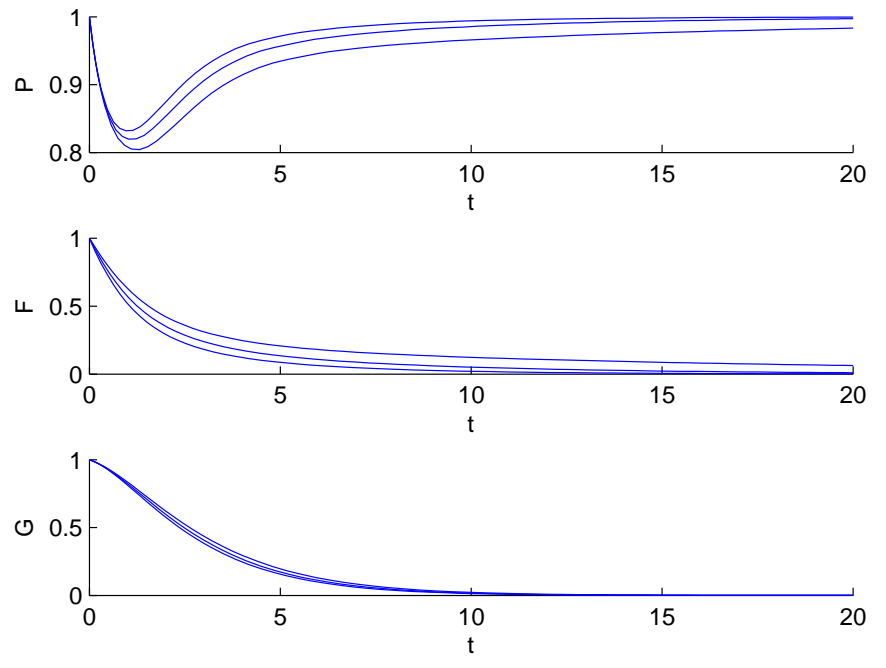

Figure 1: Time series of the solutions trajectories of (2) for the parameter values $\lambda=0.4$, $a=0.5, n=0.5, e=0.5$, and $m \in[0.31,0.51]$.

Proposition 3 The system (2) exhibits a transcritical bifurcation for $\rho=1$, involving equilibria $E_{1}$ and $E_{2}$. Furthermore, for $\rho=\frac{1}{\sqrt{3}}$ the system undergoes a supercritical Hopf bifurcation at the equilibrium $E_{2}$.

Proof. It is easy to check the first claim, as the stability condition for $E_{1}$ (9) and the feasibility condition for $E_{2}$ (4) are the opposite of one another. For the second claim, we remark that (2) is a polynomial differential system in $\mathbf{R}^{3}$, [27] and observe that two of the roots of the characteristic equation of the Jacobian evaluated at $E_{2}$ become pure imaginary for $\rho=3^{-\frac{1}{2}}$, while the third eigenvalue does not vanish in general, namely

$$
x_{1}=\frac{\lambda}{a}\left[\frac{2}{3 \sqrt{3}}-h\right] \neq 0, \quad x_{2,3}= \pm \sqrt{\frac{2 m}{3}} i .
$$

Also the transversality condition holds, as the derivative of the real part of the complex conjugate roots does not vanish for the critical value of the bifurcation parameter $\rho$. We find in fact

$$
\frac{d R e\left(x_{2,3}\right)}{d \rho}=-3 \rho,\left.\quad \frac{d \operatorname{Re}\left(x_{2,3}\right)}{d \rho}\right|_{\rho=\frac{1}{\sqrt{3}}}=-\sqrt{3} \neq 0
$$

Hence, the second claim. 


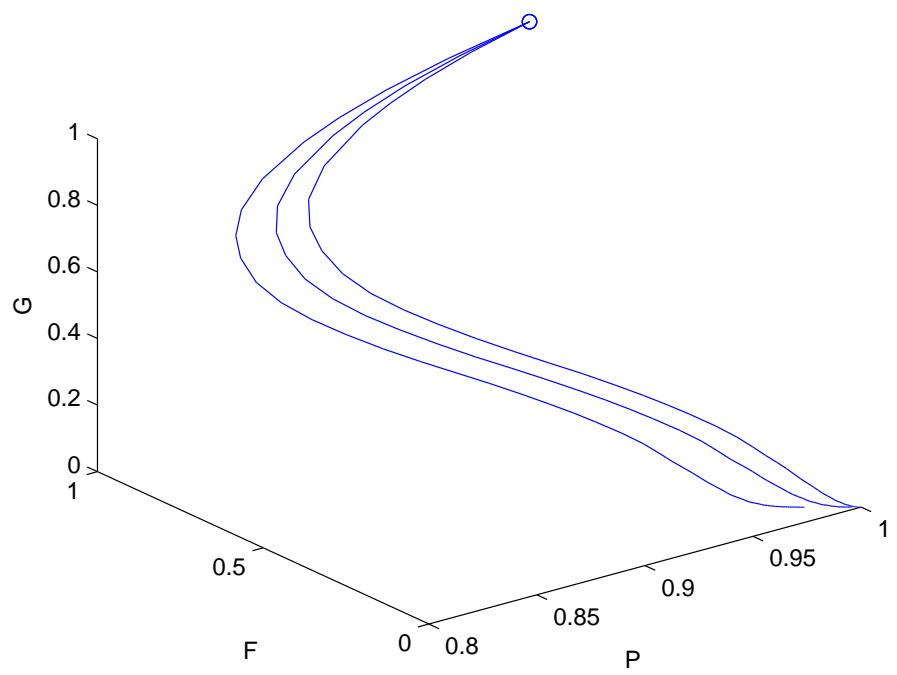

Figure 2: Phase space portrait of the solutions trajectories of (2) for the same parameter values of Figure 1.

Proposition 4 At endemic coexistence, equilibrium $E_{3}$, the system (2) admits Hopf bifurcations.

Proof. We can follow closely the ideas of [27] and impose that the point with $P_{3}=0.75$ gives the prey population at the endemic coexistence equilibrium. It follows then

$$
F_{3}=\frac{h}{a}=\frac{n}{\lambda}=\frac{21}{64 a}, \quad G_{3}=\frac{a e}{\lambda}\left(\frac{3}{4}-\rho\right), \quad h=\frac{21}{64},
$$

feasible for $\rho>0.75$. The first two Routh-Hurwitz conditions on the characteristic equation (11) become:

$$
a_{1}=\frac{11}{16}>0, \quad a_{3}=-\frac{11}{16} \times \frac{21}{64} e \lambda\left(\frac{3}{4}-\rho\right)>0 .
$$

Furthermore,

$$
a_{2}=a e\left(\frac{3}{4} n-\rho n+h\right) .
$$

The last condition, if we force it to be zero, $a_{1} a_{2}=a_{3}$, gives the critical value 

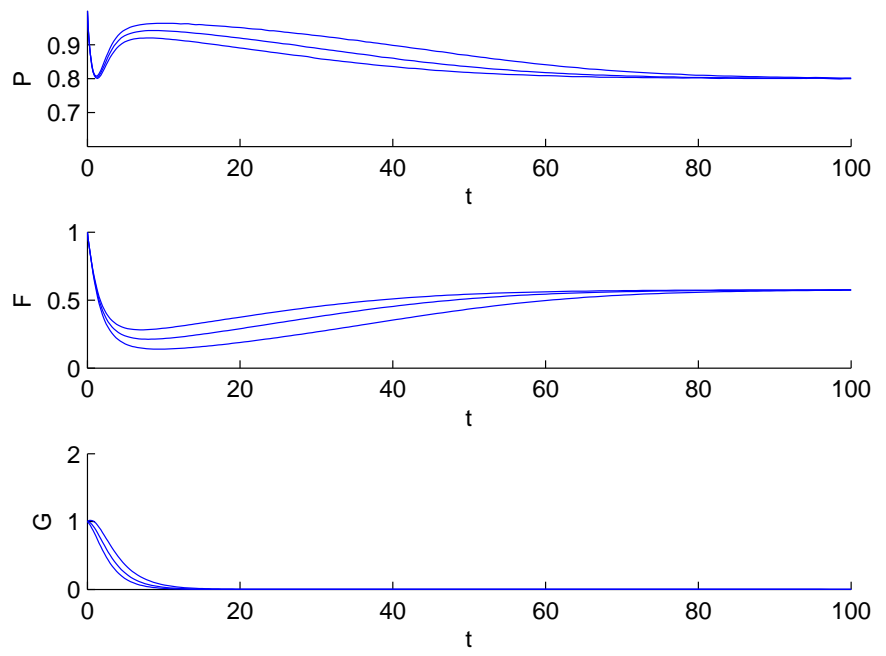

Figure 3: Time series of the solutions trajectories of (2) for the parameter values $\lambda=0.5$, $a=0.5, m=0.2, e=0.5$, and $n \in[0.41,0.61]$.

of the bifurcation parameter, which we choose here to be $\rho$ :

$$
\rho^{\dagger}=\frac{64 h}{(21+64 h) n}\left[\frac{3}{4} n+\frac{63}{256} \frac{n}{h}+h\right] .
$$

The conditions for a Hopf bifurcation in this situation are thus verified when $\rho>\rho^{\dagger}$.

Remark 4. We have obtained several simulations showing limit cycles around $E_{3}$, by varying the parameter values. However the amplitude of the oscillations is always small. We show in Figure 7 one of these results, for the parameter values $\lambda=23.8, m=0.01, n=0.3, e=0.99$ and $a=26.0313$, providing complex conjugate eigenvalues with not too small real part, namely $0.8780 \pm 0.9271 i$ and the equilibrium $E_{3}=(0.7500,0.0126,0.8117)$.

We now discuss the feasibility of these equilibria in the $\rho-h$ parameter space. The feasibility regions for $E_{1}$ is easy to draw, namely it is the half plane $\rho>1$, independently of $h$, and for $E_{2}$ we have the stability region $3^{-\frac{1}{2}}<\rho<1$ with $h>\rho\left(1-\rho^{2}\right)$, see Figure 8 . In it, note that the boundary separating the regions for $E_{2}$ and $E_{3}$ is given by the curve $h=\rho\left(1-\rho^{2}\right)$, which is part of a cubic. For $E_{3}$, we certainly need $\rho<1$ and $h<\frac{2}{9} \sqrt{3}$. Since $E_{3}$ solves the cubic (6), and for both feasibility and stability we need 


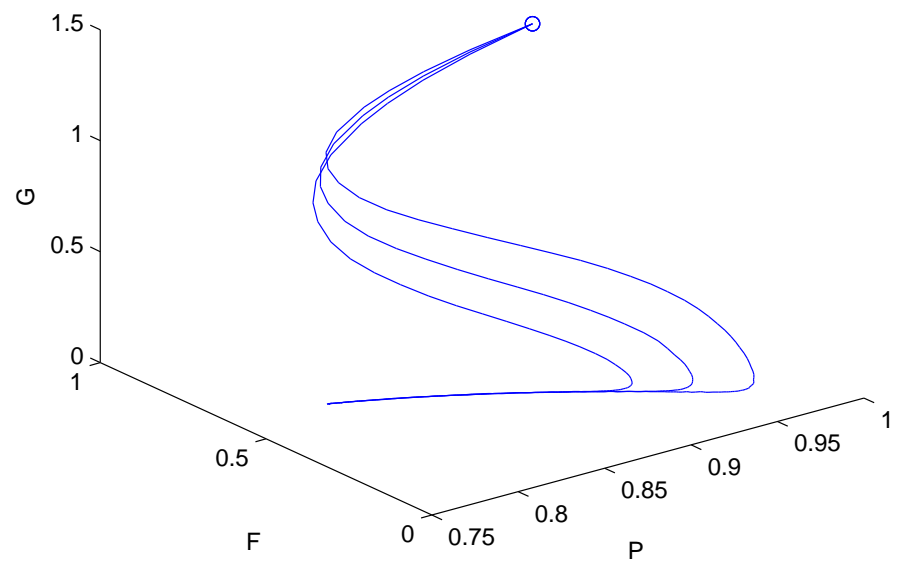

Figure 4: Phase space portrait of the solutions trajectories of (2) for the same parameter values of Figure 3.

$P_{3}>\max \left\{3^{-\frac{1}{2}}, \rho\right\}$, we have two cases. In case $\rho<3^{-\frac{1}{2}}, 3^{-\frac{1}{2}}<P_{3}$ holds, since one of the roots of (6), namely $P_{3}^{+}$, is always larger than $3^{-\frac{1}{2}}$, since the latter value corresponds to a negative minimum of the cubic. Conversely, we must have $P_{3}>\rho>3^{-\frac{1}{2}}$ and from the fact that $P_{3}$ solves the cubic (6) we have the estimate $0=P_{3}^{3}-P_{3}+h>\rho^{3}-\rho+h$ from which $h<\rho\left(1-\rho^{2}\right)$. These two results thereby identify the stability region for $E_{3}$, see Figure 8.

Considering now the parameter $\rho$ as it changes from values larger than 1 to values lower than $1, E_{1}$ and $E_{2}$ exchange their stability properties, the former also becoming feasible, so that we have the transcritical bifurcation identified earlier.

Consider now $3^{-\frac{1}{2}}<\rho<1$. In this region, we study the system as $h$ moves from above the critical curve $h=\rho-\rho^{3}$ to values below it. Initially, $E_{2}$ is feasible and stable. $E_{3}$ depends on the cubic (6) which has only one negative root, thus is unfeasible, but it would be stable if $\rho<3^{-\frac{1}{2}}$, since the stability condition $P_{3}>3^{-\frac{1}{2}}$ is obtained from $P_{3}^{2}<3^{-1}$ and $P_{3}>0$. As $h$ crosses the critical curve, $E_{2}$ becomes unstable and two new equilibria arise, of which only one is stable, while the former unfeasible equilibrium remains stable. We obtain a situation very similar to a fork bifurcation, an imperfect fork bifurcation, [28, 29, 30]. 

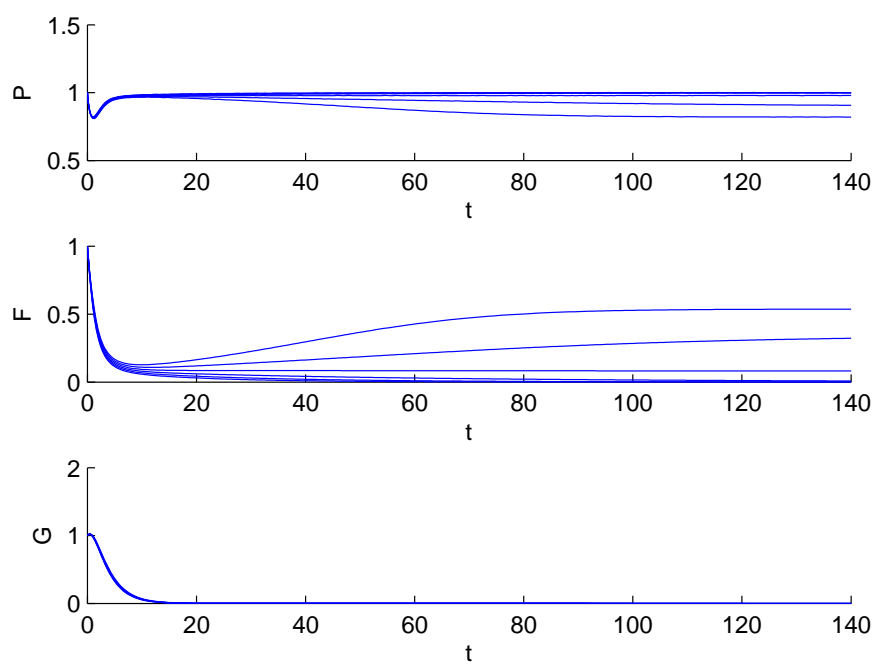

Figure 5: Time series of the solutions trajectories of (2) for the parameter values $\lambda=0.5$, $a=0.5, n=0.4, e=0.5$, and $m \in[0.205,0.305]$. Note the different equilibrium point attained as $m$ changes its value.

The behavior on the boundaries between the regions of Figure 8 falls into the following cases

1. $h=\frac{2 \sqrt{3}}{9}$ and $0<\rho<\frac{1}{\sqrt{3}}: P_{3}=\frac{1}{\sqrt{3}}$ has multiplicity 2 , so that $E_{3}=$ $\left(\frac{1}{\sqrt{3}}, \frac{h}{a}, \frac{-\sqrt{3} m+e a}{\lambda \sqrt{3}}\right)$. One of the eigenvalues of $E_{3}$ is $\mu_{1}=0$.

2. $h=\rho\left(1-\rho^{2}\right)$ and $\frac{1}{\sqrt{3}}<\rho<1: E_{2}=\left(\rho, \frac{h}{a}, 0\right)$. One of the eigenvalues of $E_{2}$ is $\mu_{1}=0$.

3. $\rho=\frac{1}{\sqrt{3}}$ and $h>\frac{2 \sqrt{3}}{9}: E_{2}=\left(\frac{1}{\sqrt{3}}, \frac{2 \sqrt{3}}{9 a}, 0\right)$. One of the eigenvalues of $E_{2}$ is real and always negative, the other two are pure imaginary numbers.

4. $\rho=1: E_{2}=(1,0,0)=E_{1}$ and one of the eigenvalues of $E_{1}$ is $\mu_{1}=0$.

5. $\rho=\frac{1}{\sqrt{3}}$ and $h=\frac{2 \sqrt{3}}{9}: P_{3}=\frac{1}{\sqrt{3}}$ with multiplicity 2 , so that $E_{3}=$ $\left(\frac{1}{\sqrt{3}}, \frac{2 \sqrt{3}}{9 a}, 0\right)=E_{2}$. One of the eigenvalues of $E_{3}$ is $\mu_{1}=0$.

Remark 5. In all of them, the behavior depends strongly on the initial conditions. For instance, taking the initial condition $(0.5,0.3,0.1)$ and the parameter values $\lambda=0.18, a=0.4, m=0.069, n=0.1 \sqrt{3}, e=0.3$ giving $\rho=0.575 \cong 3^{-\frac{1}{2}}$ and $h=2 \sqrt{3} / 9 \cong 0.38$, i.e. the above case 5 , we get the persistent oscillations behavior of Figure 9. Its phase space representation is plotted in Figure 10. 


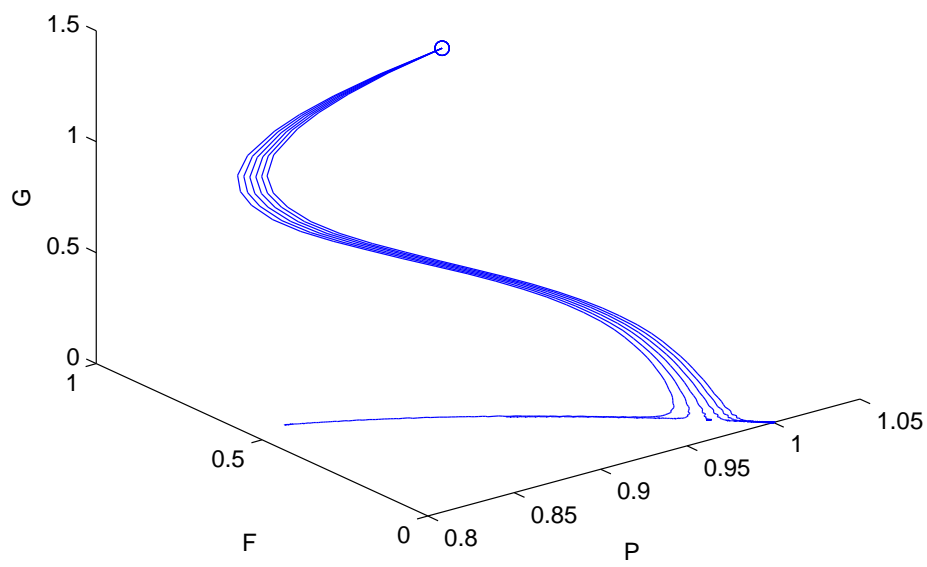

Figure 6: Phase space portrait of the solutions trajectories of (2) for the same parameter values of Figure 5.

Remark 6. Instead for the initial condition $(0.57,0.96,0.001)$, very close to the former one, we find the behavior of Figure 11 with phase space behavior in Figure 12, giving again a limit cycle, with trajectories tending to it from within. Note that persistent oscillations are not uncommon in epidemiology, [31].

Remark 7. In case 3 the presence of purely imaginary eigenvalues suggests the presence of a Hopf bifurcation for $\rho^{\ddagger}=3^{-\frac{1}{2}}$. For the parameter values $\lambda=0.1, a=0.1 \sqrt{3}, m=0.02, n=0.3, e=0.2$, giving $\rho=3^{-\frac{1}{2}}$ and $h \cong 0.51$ we have a limit cycle as shown in Figures 13 and 14 .

\section{Discussion}

We have presented a simple ecoepidemic model for predator-prey interactions in which the prey gather together in herds and a disease affects the predators. There are only three possible stable equilibrium states. The predators are wiped out, $E_{1}$, the disease is eradicated and prey and predators both thrive, $E_{2}$, and the disease remains endemic, with the three subpopulations coexisting, $E_{3}$. The relevant role of the parameter $\rho,(3)$, is apparent in this context as it has been originally discovered in [17], and in adimen- 


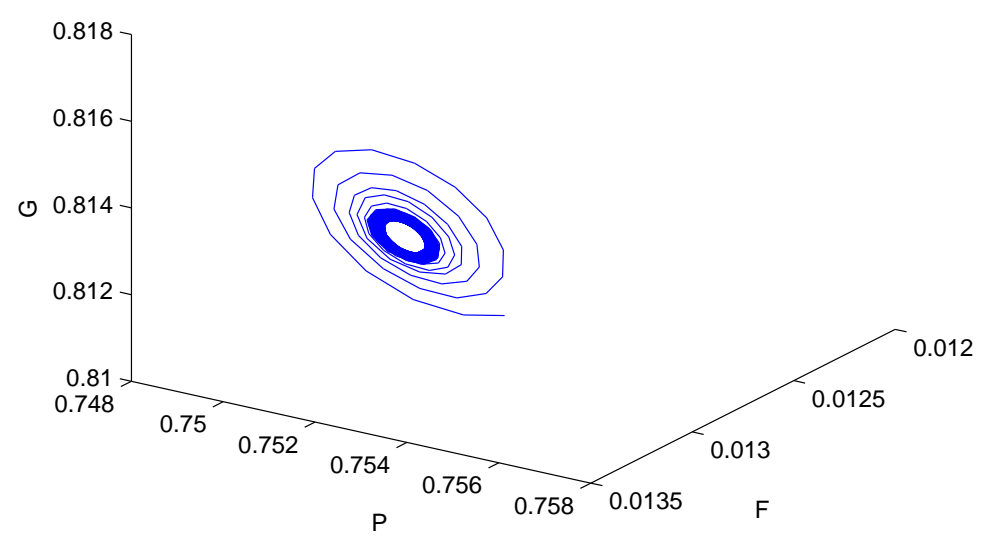

Figure 7: Tiny persistent oscillations around the endemic coexistence equilibrium $E_{3}$ for the parameter values $\lambda=23.8, m=0.01, n=0.3, e=0.99$ and $a=26.0313$.

sionalized form in [18], using a different rescaling. However, the parameter $\rho$ of $[17,18]$ has a different definition in the ecoepidemic context. We have used the same letter here, to conform ourselves to what already used in [21], in which the parameter $\rho$ is defined in terms also of the predation rate $a$. In any case, $\rho$ discriminates among all the possible dynamics of the system. It contains almost all the relevant information needed to assess the ultimate behavior of the system. Interestingly enough, its ranges are the same discovered already in [18]; those in [17] differ instead due to the different definition of $\rho$, stemming from the fact that the system has not been adimensionalized. And again, the same range appears in [21].

In summary, for $\rho>1$ the predators are wiped out, a result which agrees with what found when the disease affects the prey, [21]. For the critical value of the parameter, $\rho=1$ a transcritical bifurcation occurs and the predators establish themselves permanently in the ecosystem. For further decreasing values and also with an essential role played by the parameter $h$, also the disease becomes endemic. The three subpopulations coexist either at the stable equilibrium, bottom left blue region in Figure 8. Note that the top left white region in Figure 8 corresponds to the unfeasible equilibrium $E_{1}^{-}=(-1,0,0)$. Persistent oscillations have been discovered, in suitable 


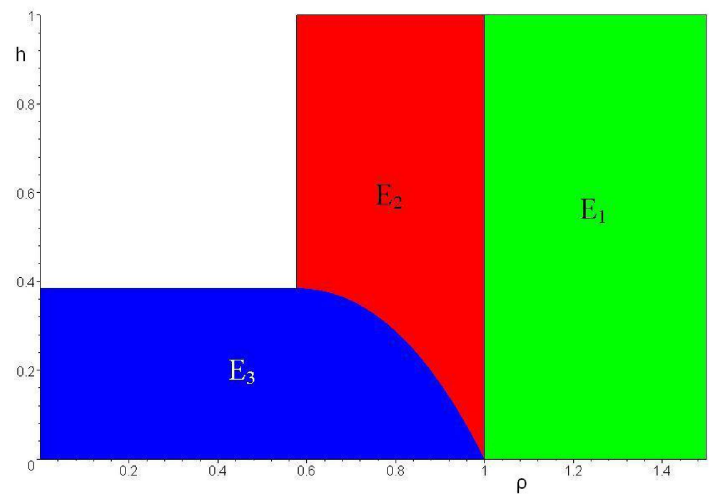

Figure 8: Feasibility and stability regions for the equilibria of the system in the $\rho-h$ parameter space.

circumstances, at the boundaries of the stability regions, and proven to exist also around the endemic coexistence equilibrium.

Note that in comparison with the other ecoepidemic case [21], when the prey are disease affected, here we have been able to better characterize the behavior of the system, the essential reason being the simpler form that the stability conditions for the coexistence equilibrium have. The background for that is the fact that the cubic characteristic equation (11) takes in this case a more workable mathematical expression.

All these remaks strongly support the claim that the ultimate outcome of the system depends mainly on $\rho$, while a smaller role is due to $h$. But while in the definition of the former only demographic-related parameters appear, in $h$ also the disease transmission is present. Thus our major biological conclusion consists in the finding that in this ecoepidemic model with group defense also the disease has some relevant influence, and therefore the outcome of the trajectories of the system is not prescribed just by demographical considerations. These considerations are in line with the standard results in ecoepidemic theory, [12, 13, 14, 15, 32, 33, 34].

Acknowledgments: The authors thank the referees for their very constructive criticism. 

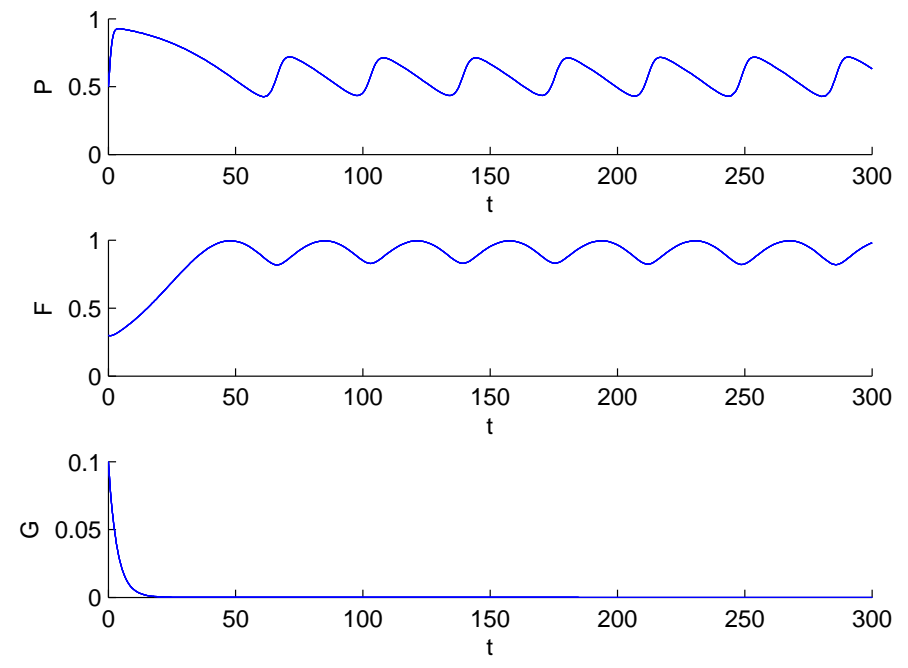

Figure 9: Population values as functions of time exhibit persistent oscillations for $\lambda=0.18$, $a=0.4, m=0.069, n=0.2 \sqrt{3}, e=0.3$ giving $\rho=0.575 \cong \frac{1}{\sqrt{3}}$ and $h=\frac{2 \sqrt{3}}{9} \cong 0.77$ and initial condition $(0.5,0.3,0.1)$.

\section{References}

[1] T. R. Malthus, An essay on the principle of population, J. Johnson in St. Paul's Churchyard, London, 1798.

[2] P. F. Verhulst, Notice sur la loi que la population suit dans son accroissement, Correspondance Mathématique et Physique Publiée par A. Quételet, 10 (1838) 113-121.

[3] A. J. Lotka, Elements of Mathematical Biology, Dover, New York, 1956.

[4] V. Volterra, U. D'Ancona, La concorrenza vitale tra le specie nell'ambiente marino, VIIe Congr. int. acqui. et de pêche, Paris (1931) $1-14$.

[5] P. Waltman, Competition models in population biology, SIAM, Philadelphia, 1983.

[6] W. O. Kermack, A. G. McKendrick, A Contribution to the Mathematical Theory of Epidemics, Proceedings of the Royal Society of London A 115 (1927) 700-721. 


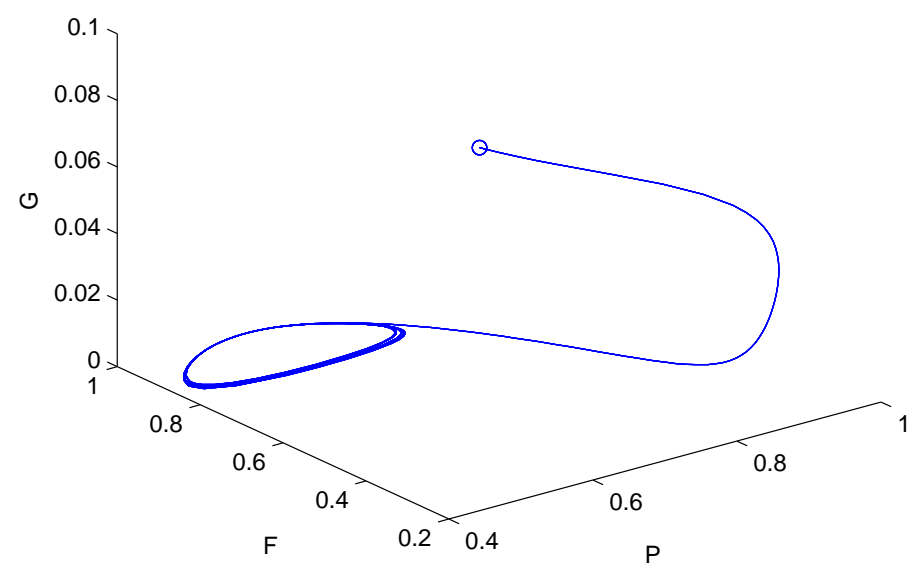

Figure 10: Phase space representation of the limit cycle, situation of Figure 9.

[7] H. von Foerster, Some remarks on changing populations, in F. Stohlmann (Ed.) The kinetics of Cellular Proliferation, Grune and Stratton, New York, 382-407, 1959.

[8] S. Busenberg, P. van den Driessche, Analysis of a disease transmission model in a population with varying size, J. of Math. Biology 28 (1990) 257-270.

[9] L. Q. Gao, H.W. Hethcote, Disease transmission models with densitydependent demographics, J. of Math. Biology 30 (1992) 717-731.

[10] J. Mena-Lorca, H. W. Hethcote, Dynamic models of infectious diseases as regulator of population sizes, J. Math. Biology 30 (1992) 693-716.

[11] K. P. Hadeler, H. I. Freedman, Predator-prey populations with parasitic infection, J. of Math. Biology 27 (1989) 609-631.

[12] E. Beltrami, T. O. Carroll, Modelling the role of viral disease in recurrent phytoplankton blooms, J. Math. Biol. 32 (1994) 857-863.

[13] E. Venturino, The influence of diseases on Lotka-Volterra systems, Rocky Mountain Journal of Mathematics 24 (1994) 381-402. 

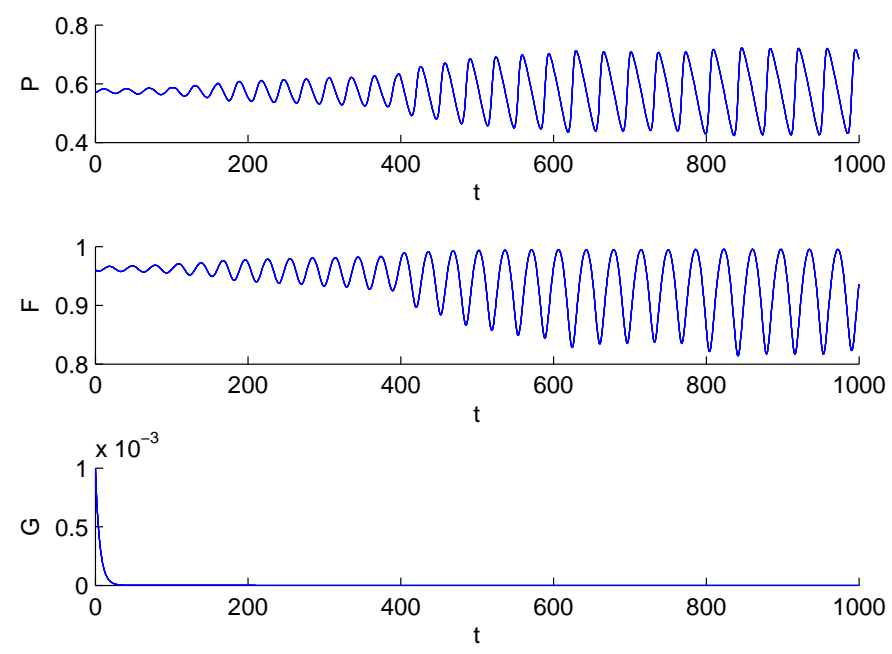

Figure 11: Population values as functions of time exhibit persistent oscillations for $\lambda=$ $0.18, a=0.4, m=0.069, n=0.2 \sqrt{3}, e=0.3$ giving $\rho=0.575 \cong \frac{1}{\sqrt{3}}$ and $h=\frac{2 \sqrt{3}}{9} \cong 0.77$ and initial condition $(0.57,0.96,0.001)$.

[14] E. Venturino, Epidemics in predator-prey models: disease among the prey, in O. Arino, D. Axelrod, M. Kimmel, M. Langlais: Mathematical Population Dynamics: Analysis of Heterogeneity, Vol. one: Theory of Epidemics, Wuertz Publishing Ltd, Winnipeg, Canada, p. 381-393, 1995.

[15] H. Malchow, S. Petrovskii, E. Venturino, Spatiotemporal patterns in Ecology and Epidemiology, CRC, Boca Raton, 2008.

[16] H. I. Freedman, G. Wolkowitz, Predator-prey systems with group defence: the paradox of enrichment revisited, Bull. Math. Biol. 48 (1986) 493-508.

[17] V. Ajraldi, E. Venturino, Mimicking spatial effects in predator-prey models with group defense, in J. Vigo Aguiar, P. Alonso, S. Oharu, E. Venturino, B. Wade (Eds), Proceedings of the International Conference CMMSE 2009, v. 1 (2009) 57-66.

[18] V. Ajraldi, M. Pittavino, E. Venturino, Modelling herd behavior in population systems, Nonlinear Analysis: Real World Applications 12(4) (2011) 2319-2338. 


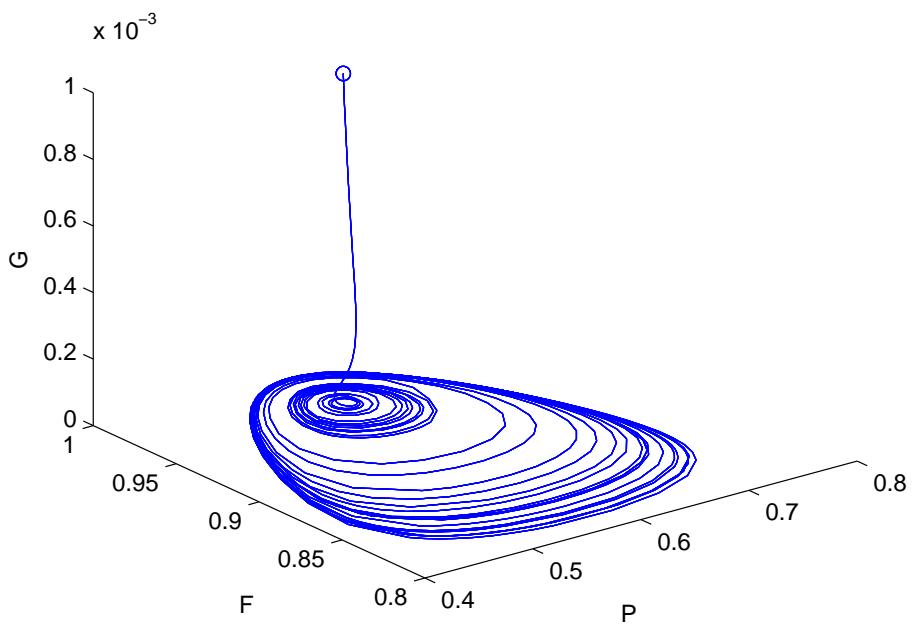

Figure 12: Phase space representation of the limit cycle on the $P F$ plane, situation of Figure 11.

[19] P. A. Braza, Predator-prey dynamics with square root functional responses, Nonlinear Analysis Real World Applications 13(4) (2012) 18371843.

[20] C. Cosner, D. L. DeAngelis, J. S. Ault, D. B. Olson, Effects of spatial grouping on the functional response of predators, Theoretical Population Biology 56 (1999) 65-75.

[21] E. Venturino, A minimal model for ecoepidemics with group defence, Journal of Biological Systems 19(4) (2011) 763-785.

[22] G. Birkhoff, G.-C. Rota, Ordinary differential equations, 3rd Edition, Wiley, New York, 1978.

[23] S. Belvisi, N. Tomatis, E. Venturino, Ecoepidemic models with identifiable infectives I: disease in the prey, Proceedings of the 10th International Conference on Computational and Mathematical Methods in Science and Engineering, CMMSE 2010, Almeria, Spain, June 27th-30th, 2010, v. 1 (2010) p. 188-198. 

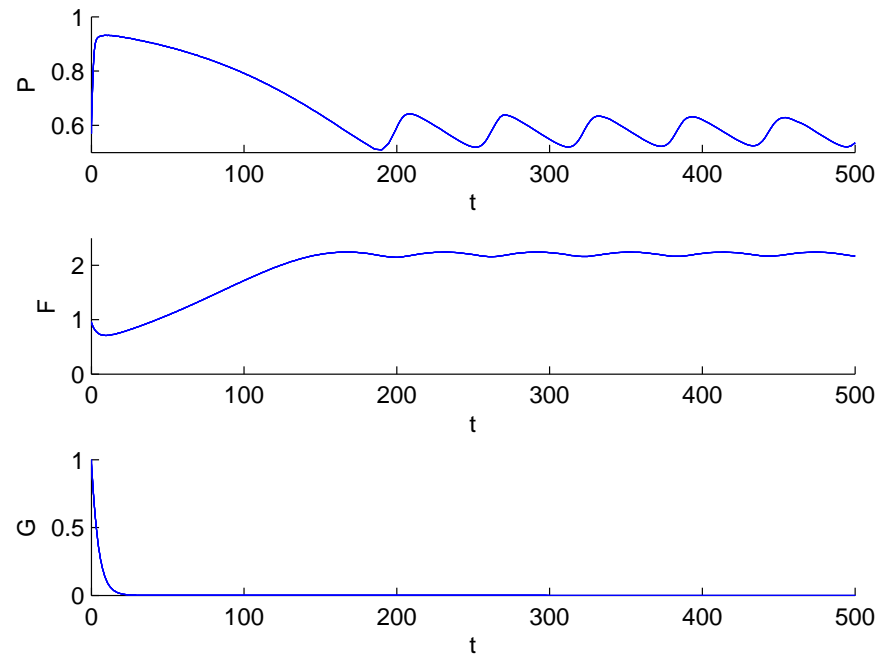

Figure 13: Population values as functions of time exhibit persistent oscillations for $\lambda=0.1$, $a=0.1 \sqrt{3}, m=0.02, n=0.3, e=0.2$, giving $\rho=\frac{1}{\sqrt{3}}$ and $h \cong 0.51$.

[24] S. Belvisi, N. Tomatis, E. Venturino, Ecoepidemics with Identifiable Infectives II: Disease in the Predators, ICNAAM 2010: International Conference of Numerical Analysis and Applied Mathematics 2010. AIP Conference Proceedings, v. 1281 (2010) 743-746.

[25] E. Venturino, Epidemics in predator-prey models: disease in the predators, IMA Journal of Mathematics Applied in Medicine and Biology 19 (2002) 185-205.

[26] F.M.D. Gulland, (1995), The impact of infectious diseases on wild animal populations - a review, in Ecology of infectious diseases in natural populations, (B.T. Grenfell, A.P. Dobson Editors), Cambridge Univ. Press, p. 20-51.

[27] M. Falconi J. Llibre, Hopf bifurcation in predator-prey models with an age structured prey, in BioMat2011, International Symposium on Mathematical and Computational Biology, R. P. Mondaini (Editor), World Scientific, p. 11-22, 2012. 


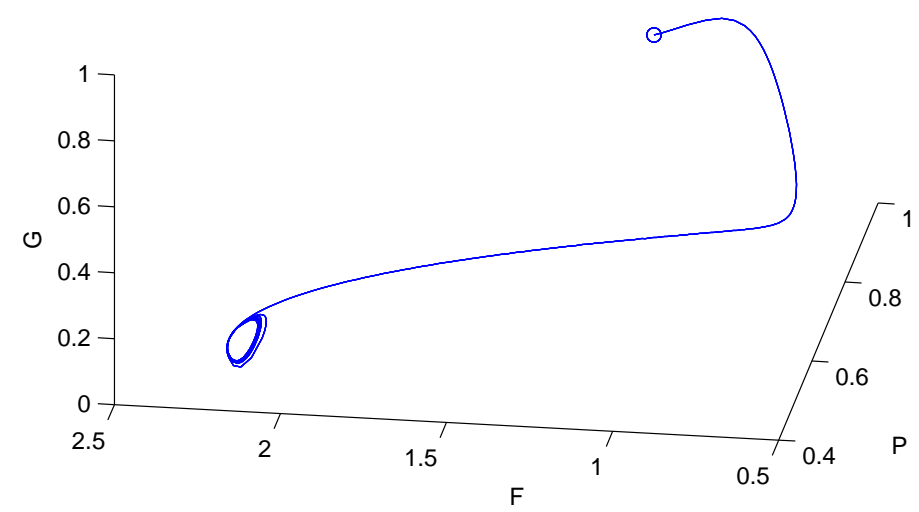

Figure 14: Phase space representation of the limit cycle, situation of Figure 13.

[28] J. K. Hale, H. Koçak, Dynamics and bifurcations, Springer, New York, 1991.

[29] L. Perko, Differential equations and dynamical systems, 3rd ed., Springer-Verlag, New York, 2001.

[30] R. Riganti, Biforcazioni e caos nei modelli matematici delle scienze applicate (Bifurcations and chaos in mathematical models of applied sciences), Levrotto \& Bella, Torino 2000.

[31] P. Manfredi, E. Salinelli, Population-induced oscillations in blended SISEI epidemiological models, IMA J. Math. Appl. Med. Biol. 19 (2002) 95-112.

[32] E. Venturino, The effects of diseases on competing species, Math. Biosc. 174 (2001) 111-131.

[33] M. Haque, E. Venturino, The role of transmissible diseases in HollingTanner predator-prey model, Theoretical Population Biology 70 (2006) 273-288. 
[34] M. Haque, E. Venturino, An ecoepidemiological model with disease in the predator; the ratio-dependent case, Math. Meth. Appl. Sci. 30 (2007) 1791-1809. 